Z. Epileptol. 2022 · 35:36-41

https://doi.org/10.1007/s10309-021-00463-8

Angenommen: 22. Dezember 2021

Online publiziert: 14. Januar 2022

(c) Der/die Autor(en) 2022

\section{Invasive Diagnostik und resektive Chirurgie bei Frontallappenepilepsie}

\author{
Daniel Delev · Hans Clusmann \\ Klinik für Neurochirurgie, Universitätsklinikum RWTH Aachen, Aachen, Deutschland
}

\title{
Zusammenfassung
}

Frontallappenepilepsie stellt das häufigste extratemporale Epilepsiesyndrom dar und somit auch die zweithäufigste fokale Epilepsieform nach der Temporallappenepilepsie. Die nicht immer lokalisierenden elektrophysiologischen Befunde, welche schnell über die Insel oder den Balken geleitet werden können, sowie das häufige Fehlen einer sichtbaren epileptogenen Läsion machen die Anwendung invasiver Abklärung mittels Tiefen- und/oder subduralen Elektroden in vielen Fällen unabdingbar. Das Spektrum der chirurgischen Resektionen im Bereich des Frontallappens reicht von einfachen fokalen erweiterten Läsionektomien über Frontallappendiskonnektionen bis hin zur frontalen Lobektomie. Entsprechend variiert auch das postoperative epileptologische Outcome mit Anfallsfreiheitsraten zwischen 20\% und 70\%. Die vorliegende Arbeit gibt eine Übersicht über die Möglichkeiten der invasiven Abklärung und der operativen Strategien bei Frontallappenepilepsie. Zusätzlich werden die postoperativen Ergebnisse und zugrunde liegende Einflussfaktoren zusammengefasst. Insgesamt bleibt die Frontallappenepilepsie eine epilepsiechirurgische Herausforderung und somit auch die Hoffnung, dass zukünftige Fortschritte im Bereich der Bildgebung oder Neuromodulation zu einer Verbesserung der postoperativen Ergebnisse beitragen können.

\section{Schlüsselwörter}

Therapierefraktär · MRT-negativ · Outcome · Prädiktive Faktoren · Neuromodulation

Etwa $25-40 \%$ der Patienten mit Epilepsie leiden unter medikamentös refraktären Anfällen. Dies kann zu kognitiven Beeinträchtigungen, neuropsychologischen Folgeerscheinungen, verminderter Lebensqualität und erhöhter Mortalität führen $[2,25,38,43]$. Die chirurgische Therapie ist eine sichere und anerkannte Behandlungsoption für Patienten mit fokaler, therapierefraktärer Epilepsie mit guten Erfolgsraten, die höchste Evidenz besteht für temporomesiale Epilepsien [44].

Die Frontallappenepilepsie ist nach der Temporallappenepilepsie die zweithäufigste fokale Epilepsieform. Sie stellt mit 20-40\% aller fokalen Epilepsien gleichzeitig das häufigste extratemporale Epilepsiesyndrom dar [39].

Die Frontallappenepilepsien werden entsprechend der Lokalisation der epi- leptogenen Zone in laterale, mesiale und basale Frontallappenepilepsien eingeteilt. Die lateralen frontalen Bereiche werden in einen präfrontalen, prämotorischen und zentralen (rolandischen) Bereich unterteilt, welche 3 funktionell wichtige Bereiche enthalten - 1) beidseitig den primären motorischen Kortex, und 2) das supplementäre motorische Areal (SMA) sowie 3) die Broca-Sprachregion der dominanten Hemisphäre. Entsprechend dieser funktionellen Anatomie zeigen epileptische Anfälle, die im zentralen Frontallappen ihren Ursprung haben, charakteristische somatosensorische Auren oder fokale motorische Entäußerungen mit tonischen, klonischen oder komplexen motorischen Symptomen. Wenn die Broca-Sprachregion involviert ist, können epileptische Anfälle typischerweise mit einer Aphasie oder Dysphasie einhergehen [17]. 

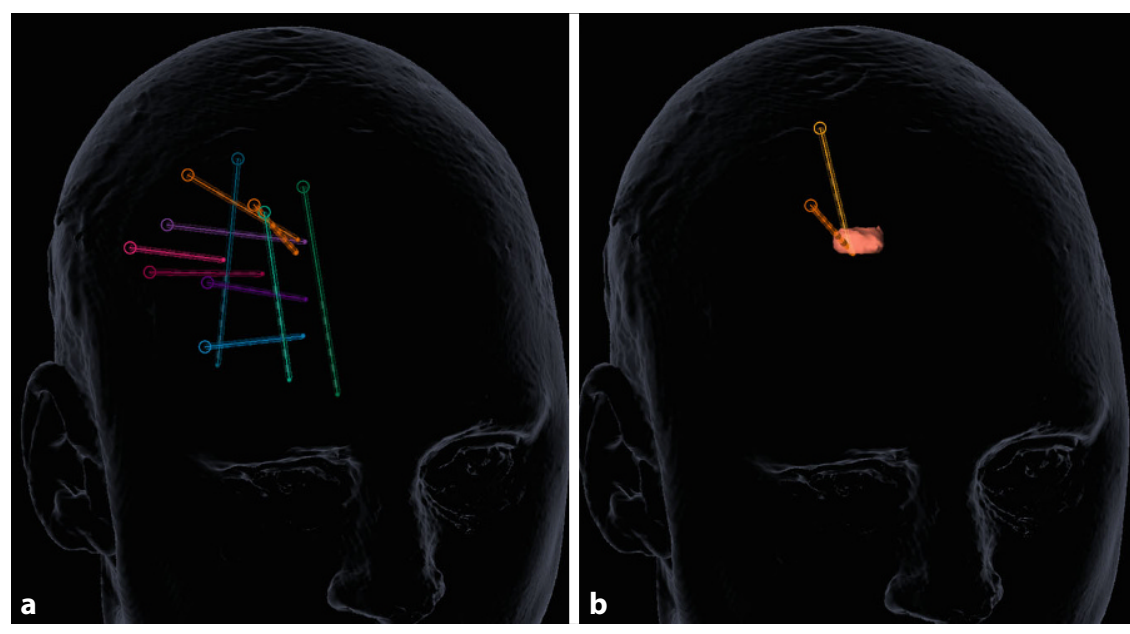

Abb. 1 ॥ Beispielhafte schematische Darstellung der Möglichkeit einer invasiven Abklärung des Frontallappens. a MRT-negative Epilepsie, die Abklärung beinhaltet 3 orthogonale Elektroden, welche F1, F2, Frontobasis und Insel ableiten, sowie mehrere Elektroden von lateral, welche F2 und F3 ableiten. b Implantation von nur 2 Elektroden nach multimodaler bildgebender Diagnostik, welche die epileptogene Zone möglichst genau lokalisiert hat. Die invasive Ableitung hat hier primär das Ziel, die mutmaßliche epileptogene Zone zu bestätigen oder zu verwerfen

Hypermotorische Anfälle sind ebenfalls typische im Frontallappen entstehende Anfälle, die aus dem prämotorischen und präfrontalen Kortex entstehen [21]. Typische in der SMA entstehende Anfälle sind z. B. bilaterale asymmetrische tonische Anfälle mit einem raschen Begin mit tonischer Körperhaltung, Abduktion und Beugung der oberen Extremitäten sowie Abduktion und Halbbeugung der unteren Extremitäten [5].

Die semiologische Charakterisierung der Anfälle mit Ursprung im basalen Frontallappen stellt eine der größten Herausforderungen im Rahmen der Epilepsiediagnostik dar. Der basale Frontallappen bleibt während des Anfallsbeginns erfahrungsgemäß klinisch stumm [36], und die klinische Präsentation des Anfalls entspricht somit einer Reizung anderer funktioneller Areale [31]. Dazu noch ist der basale Frontallappen über mehrere subkortikale Projektionen mit dem lateralen Frontallappen, dem insulären Kortex und über den Uncus auch mit dem Temporallappen verbunden. Dementsprechend können diese Kortexareale semiologisch über eine schnelle Propagation an der Anfallssemiologie beteiligt sein [16]. Zusammenfassend ist die Semiologie der Frontallappenepilepsie vielfältig, und somit sind die Bemühungen um eine präzise Lokalisation und Klassifizierung mit großen Herausforderungen verbun- den, welche in vielen Fällen nur durch die Anwendung invasiver Diagnostik mit Implantation unterschiedlicher Tiefenoder subduralen Elektroden gelöst werden können. Die aktuelle Arbeit stellt eine narrative Übersichtsarbeit über die Diagnostik und chirurgische Behandlung der Frontallappenepilepsie dar und versucht unterschiedliche chirurgische Optionen sowie postoperative Ergebnisse zusammenzufassen.

\section{Invasive Diagnostik bei Frontallappenepilepsien}

Die prächirurgische Abklärung einer Frontallappenepilepsie stellt häufig eine diagnostische Herausforderung dar. Die Schwierigkeit der genauen Lokalisation eines frontalen Anfallsursprunges schlägt sich in der stark variierenden postoperativen Anfallsfreiheit von $20 \%$ bis zu $70 \%$ nieder. Einer der wichtigsten Gründe dafür ist die hohe Zahl an Patienten, die keine spezifische epileptogene Läsion aufweisen. Unterschiedliche nichtinvasive Verfahren sowie deren Kombinationen können die Lokalisation der epileptogenen Zone in MRT-negativen Patienten verbessern. So konnte die Arbeit von Moeller et al. zeigen, dass die Kombination aus EEG, fMRT, SPECT und PET die Lokalisierung der epileptogenen Zone positiv beeinflussen kann [30]. Auch die Einfüh- rung der MAP-Analyse (Morphometric Analysis Programm) hat die Detektionsrate kleiner FCDs erhöht $[11,19]$. Trotzdem bleibt eine Vielzahl an Patienten ohne eine wegweisende Läsion und benötigt somit eine weiterführende invasive Abklärung ([4]; - Abb. 1).

Grundsätzlich existieren 2 Hauptstrategien, um die Planung und Implantation von Elektroden zur invasiven Abklärung des Frontallappens durchzuführen. Dieerste Möglichkeit ist eine umfangreiche invasive Diagnostik mit mehreren Tiefenoder subduralen Elektroden, welche das Ziel haben, die vermutete epileptogene Zone zu charakterisieren. Die zweite Option ist die Anwendung weniger Elektroden, welche eine bereits lokalisierte epileptogene Zone nur bestätigen oder verwerfen sollen. Die zweite Strategie entspricht dem sog. "Go/No-Go"-Prinzip, bei dem die von den Elektroden gelieferten Informationen eine resektive Operation nur triggern oder verwerfen sollen. Dies erfordert allerdings eine bereits präzise Hypothese aus einer umfangreichen nichtinvasiven Diagnostik, welche alle bildgebenden, klinischen und elektrophysiologischen Aspekte kombiniert, um die vermutete epileptogene Zone möglichst genau zu lokalisieren [11].

Der Trend zur Implantation von Tiefenelektroden setzt sich fort, obwohl subdurale Grids und Streifen bei der invasiven Abklärung von Frontallappenepilepsien nach wie vor nützlich sein können. Insbesondere bei Patienten mit frontomesialer Epilepsie stellen die interhemisphärischen subduralen Streifenelektroden eine gute Möglichkeit zum Nachweis einer epileptogenen Zone dar [12]. Subdurale Elektroden können große kortikale Bereiche abdecken und sind insbesondere bei der Kartierung von Hirnarealen mit hoher Funktionalität sehr nützlich. In jüngster Zeit wurden jedoch erste Ergebnisse der kortikalen Kartierung durch direkte kortikale Stimulation unter Verwendung von Tiefenelektroden sowohl für die Motorik als auch für die Sprachfunktion veröffentlicht $[1,10,18]$.

\section{Chirurgische Behandlung von Frontallappenepilepsien}

Frontallappenresektionen unterscheiden sich in mehreren Aspekten von den chi- 
rurgisch klar definierten temporomesialen oder temporalen Resektionen v. a. durch die Tatsache, dass die epileptogene Zone unterschiedliche Kortexregionen betreffen kann. Deshalb ist die Formulierung einer Operationsstrategie oft deutlich schwieriger als bei den weitgehend standardisierten temporalen Resektionen [35] insbesondere für Patienten, die in der bildgebenden Diagnostik keine sichtbare Läsion aufweisen [3] und keine eindeutig lokalisierenden elektroenzephalographischen Befunde haben. Eine weitere große Herausforderung ergibt sich zusätzlich durch die häufig enge Beziehung des epileptogenen Areals zu eloquenten Kortexarealen (z.B. Motorik oder Sprache). Sind diese Areale Teil des vermuteten epileptogenen Areals, ist die chirurgische Behandlungsoption einschränkt, aber nicht per se ausgeschlossen.

Frontallappenresektionen umfassen komplette Lobektomien, erweiterte Läsionektomien und diskonnektive Operationen. Darüber hinaus können neuromodulative Verfahren wie die tiefe Hirnstimulation eine gute und langfristige Anfallsreduktion bei Patienten, welche nicht infrage für eine resektive Operation kommen, zeigen [37].

\section{Frontallappenabsetzung/ Frontallappendiskonnektion}

Die komplette Resektion des Frontallappens ist eines der standardisierten Verfahren für die chirurgische Behandlung der Frontallappenepilepsie. Nach festen anatomischen Orientierungspunkten kann eine Frontallappenresektion mit geringer Komplikationsrate durchgeführt werden. Wen et al. haben vor Kurzem eine Arbeit veröffentlicht, in der die einzelnen Schritte während der Operation beschrieben wurden [42]. Sie zeigen die Möglichkeiten auf, die Resektion so durchzuführen, dass die Öffnung des Vorderhorns des Seitenventrikels vermieden wird und die SMA-Region bei Bedarf geschont wird. Obwohl die Resektion der SMA-Region insbesondere in der dominanten Hemisphäre zu länger bestehenden neurologischen Defiziten führen kann, wurde in einer aktuellen Studie von Kasabeh et al. berichtet, dass sich die verschlechterte neurologische Funktion 6 Monate nach der Ope- ration vollständig erholte [24]. Allerdings beinhaltete dieStudienpopulation v. a. Kinder (Durchschnittsalter 10 Jahre, $n=23$ ), zumindest in dieser Subgruppe schienen Resektionen der SMA-Region ohne höheres Risiko für schwerwiegende verbleibende neurologische Defizite durchführbar zu sein.

Vor Kurzem wurde eine neue Technik der Frontallappendiskonnektion vorgeschlagen und detailliert beschrieben [8, 23]. Die Idee dieses Verfahrens besteht darin, die Verbindung des Frontallappens zum restlichen Gehirn zu durchtrennen und so typische Komplikationen zu vermeiden, die mit großvolumigen Resektionen verbunden sind. In einer der ersten Serien wurde über 16 Patienten berichtet, die sich diesem Verfahren unterzogen [23]. Eine Anfallsfreiheit konnte bei der Hälfte der Patienten erreicht werden. Neurologische Komplikationen traten bei $18 \%$ der Patienten auf.

\section{Frontale Läsionsektomie und erweiterte Resektionen}

Neben den bereits erwähnten großen und ausgedehnten Operationen bestehen die meisten Resektionen bei Frontallappenepilepsie aus erweiterten Läsionektomien der vermuteten epileptogenen Zone. Der oben beschriebenen Anatomie folgend, können Resektionen innerhalb des Frontallappens in 1) polar, 2) lateral/Konvexität, 3) orbitofrontal, 4) mesial frontal/SMA und 5) zentral motorisch eingeteilt werden, wobei der letzte Bereich häufig als „Rolandischer Kortex" bezeichnet und häufig als Teil der "Zentrallappenepilepsien" behandelt wird. Diese anatomische Klassifizierung [5] wurde durch eine umfassende anatomisch-elektroklinische Klassifizierung unterstützt und bestätigt. Dafür wurde eine Clusteranalyse von 54 Patienten, die während der präoperativen Untersuchung mittels invasiver EEG untersucht wurden, durchgeführt. Die Autoren fanden 4 verschiedene Cluster mit unterschiedlichen semiologischen und EEG-Merkmalen, was die Heterogenität der Frontallappenepilepsie bestätigt und auf die Bedeutung der maßgeschneiderten Resektionen hinweist. Dies wird noch deutlicher, wenn die verschiedenen anatomischen $\mathrm{Re}$ gionen in kleinere Unterregionen unter- teilt werden, welche eigene elektroklinische Repräsentationen aufweisen können. So unterteilten Unnwongse et al. die mesialen Frontallappen in 4 weitere Unterregionen: a) frontales Cingulum, b) mittleres Cingulum, c) Prä-SMA und d) SMA [40]. Diese Unterteilung scheint insbesondere dann wichtig zu sein, wenn das postoperative epileptologische Outcome herangezogen wird. Operationen innerhalb des Gyrus cinguli führten zu einer Anfallsfreiheit von $43 \%$, während eine erweiterte supracinguläre Resektion ein deutlich besseres Outcome mit $71 \%$ Anfallsfreiheit zeigte [28]. Für das Outcome nach Resektionen in der SMA-Region berichteten die Autoren von 55\% Anfallsfreiheit bei 22 Patienten [24]. Die orbitofrontale Region scheint nach epilepsiechirurgischen Eingriffen mit 70,4\% Anfallsfreiheit die zufriedenstellendsten Ergebnisse zu liefern [45]. Bei Patienten mit frontaler Epilepsie mit Beteiligung von Gyrus praecentralis sollte grundsätzlich entschieden werden, ob die Resektion den präzentralen Gyrus einschließen sollte, was ein größeres Risiko für dauerhafte neurologische Defizite birgt. Delev et al. berichteten über einen gutes epileptologisches Outcome mit $71 \%$ ILAE 1 nach Resektionen mit Beteiligung des präzentralen Gyrus. Gleichzeitig traten dauerhafte neurologische Ausfälle unterschiedlicher Schweregrade bei immerhin $31 \%$ der Patienten [13] auf.

\section{Outcome und prognostische Faktoren}

Die diagnostischen Schwierigkeiten und die Heterogenität der Operationen erklären auch, warum die Anfallsfreiheitsraten nach Resektion bei Frontallappenepilepsie zwischen $20 \%$ und $70 \%$ variieren. Im Jahr 2012 veröffentlichten Englot et al. eine Metaanalyse mit einer Zeitspanne von 20 Jahren über 1199 Patienten, welche aufgrund einer Frontallappenepilepsie operiert wurden [14]. Wichtige prognostische Faktoren, die mit einer Anfallsfreiheit einhergingen, waren läsionelle Epilepsie und Läsionsnachweis in den präoperativen MRT. Darüber hinaus waren präzise und lokalisierte Resektionen (im Vergleich zu großen und "ungezielten" Lobektomien) und die vollständige Resektion der epileptogenen Läsion ebenfalls mit einem besseren 
Tab. 1 Übersicht über Fallserien mit Frontallappenresektionen

\begin{tabular}{|c|c|c|c|c|c|c|c|c|c|}
\hline$N$ & Autor & Jahr & $\begin{array}{l}\text { Follow- } \\
\text { up (Mo.) }\end{array}$ & $\begin{array}{l}\text { Patien- } \\
\text { tenzahl }\end{array}$ & Region & $\begin{array}{l}\text { Anfalls- } \\
\text { freiheit [\%] }\end{array}$ & $\begin{array}{l}\text { Klassifi- } \\
\text { kation }\end{array}$ & $\begin{array}{l}\text { Prädiktoren Anfalls- } \\
\text { freiheit }\end{array}$ & $\begin{array}{l}\text { Prädiktoren keine } \\
\text { Anfallsfreiheit }\end{array}$ \\
\hline 1 & $\begin{array}{l}\text { Englot } \\
{[14]}\end{array}$ & 2012 & $>12$ & 1199 & Frontallappen & 45 & Engel I & $\begin{array}{l}\text { Epileptogene Läsion, } \\
\text { umschriebene Resektion, } \\
\text { komplette Resektion }\end{array}$ & - \\
\hline 2 & $\begin{array}{l}\text { Lazow } \\
{[27]}\end{array}$ & 2013 & 12 & 58 & Frontallappen & 57 & Engel I & $\begin{array}{l}\text { Keine statistisch signifi- } \\
\text { kanten Prädiktoren }\end{array}$ & - \\
\hline 3 & Delev [13] & 2014 & $>12$ & 28 & $\begin{array}{l}\text { Gyrus praecentra- } \\
\text { lis/frontodorsaler } \\
\text { Frontallappen }\end{array}$ & 71 & ILAE 1 & $\begin{array}{l}\text { Komplette Resektion, } \\
\text { spezifische Histologie, } \\
\text { jüngeres Alter, Fehlen von } \\
\text { einfach-partialen Anfällen }\end{array}$ & - \\
\hline 4 & Bonini [4] & 2017 & 12 & 42 & Frontallappen & 59 & Engel I & Komplette Resektion & - \\
\hline 5 & $\begin{array}{l}\text { Ramantani } \\
\text { [32] }\end{array}$ & 2017 & $>12$ & 75 & Frontallappen & 63 & Engel I & $\begin{array}{l}\text { Komplette Resektion, kür- } \\
\text { zere Epilepsiedauer, streng } \\
\text { regionale epileptogene } \\
\text { Entäußerungen }\end{array}$ & - \\
\hline 6 & $\begin{array}{l}\text { Samuel P } \\
{[22]}\end{array}$ & 2019 & 12 & 73 & Frontallappen & 48 & Engel I & - & $\begin{array}{l}\text { Ältere Patienten, } \\
\text { längere Epilepsie- } \\
\text { dauer, postoperative } \\
\text { ETPs }\end{array}$ \\
\hline 7 & Zhao [45] & 2021 & $>12$ & 27 & $\begin{array}{l}\text { Orbitofrontaler } \\
\text { Frontallappen }\end{array}$ & 70 & Engel I & $\begin{array}{l}\text { Keine statistisch signifi- } \\
\text { kanten Prädiktoren }\end{array}$ & - \\
\hline
\end{tabular}

epileptologischen Outcome assoziiert. Die - Tab. 1 gibt einen Überblick über Studien und Patientenserien, die nach 2012 veröffentlicht wurden und daher nicht in der Metaanalyse von Englot et al. enthalten sind. Für die Suche wurden die Begriffe "frontal lobe epilepsy surgery, seizure outcome, predictors" benutzt und dieZeitspanne zwischen 2012 und 2021 erfasst. Eine wichtige und interessante Tatsache ist, dass die meisten dieser Arbeiten über eine Anfallsfreiheit von mehr als $50 \%$ berichteten. In einzelnen Fallserien, bei denen nur spezifische Subgruppen von Frontallappenresektionen untersucht wurden, reicht die Anfallsfreiheit sogar bis zu $70 \%$. Obwohl sich die prädiktiven Faktoren nicht einheitlich in allen Studien finden, gibt es eine Reihe von ziemlich konsistenten Ergebnissen: Eine sichtbare epileptogene Läsion im MRT, eine kürzere Dauer der Epilepsie und eine vollständige Resektion der epileptogenen Läsion stellen die wichtigsten prognostischen Faktoren dar, die mit einem günstigen Anfallsergebnis nach der Operation assoziiert sind. Neben der Anfallsfreiheit stellt das neuropsychologische Outcome einen der wichtigsten Outcomeparameter dar. Hierzu konnten 2 Übersichtsarbeiten zeigen, dass eine Verschlechterung der kognitiven Funktion durchaus in $42 \%$ der operierten Patienten auftreten kann $[6,29]$. Am meisten gefährdet waren die Domäne der Intelligenz und der exekutiven Funktionen. Als Risikofaktoren wurden das hohe Patientenalter, eine fehlende MRT-Läsion und ein schlechtes Anfallsoutcome identifiziert. Bei den restlichen Patienten waren die postoperativen neuropsychologischen Ergebnisse entweder verbessert, unverändert oder zeigten ein Mischbild [29].

\section{Ausblick}

Trotz mittlerweile über 30 Jahren veröffentlichter Erfahrung bleibt die Chirurgie der Frontallappenepilepsie eine Herausforderung. Daher sind aktuelle und künftige Entwicklungen erforderlich, um sowohl das Anfallsgeschehen als auch die funktionellen Ergebnisse zu verbessern. Fortschritte in der Bildgebung und der zunehmende Einsatz von 7 T- oder 9,4 T-Hochfeld-MRT können die Entdeckungsrate epileptogener Läsionen erhöhen [41]. Zusätzlich können weitere bildgebende Verfahren Informationen über das funktionelle und strukturelle Konnektom liefern und dazu beitragen, epileptogene Netzwerke zu identifizieren [7, 15, 26]. Und schließlich werden neuartige Methoden der Neuromodulation und minimal-invasive Ansätze die neurochirurgischen Möglichkeiten be- reichern und eine Option für jene Patienten darstellen, die für eine resektive Operation nicht infrage kommen [3, 9, 20, 33, 34].

\section{Korrespondenzadresse}

\section{Daniel Delev}

Klinik für Neurochirurgie, Universitätsklinikum RWTH Aachen

Pauwelsstrae 30, Aachen, Deutschland

ddelev@ukaachen.de

Funding. Open Access funding enabled and organized by Projekt DEAL.

\section{Einhaltung ethischer Richtlinien}

Interessenkonflikt. D. Delev und H. Clusmann geben an, dass kein Interessenkonflikt besteht.

Für diesen Beitrag wurden von den Autoren keine Studien an Menschen oder Tieren durchgeführt. Für die aufgeführten Studien gelten die jeweils dort angegebenen ethischen Richtlinien.

Open Access. Dieser Artikel wird unter der Creative Commons Namensnennung 4.0 International Lizenz veröffentlicht, welche die Nutzung, Vervielfältigung, Bearbeitung, Verbreitung und Wiedergabe in jeglichem Medium und Format erlaubt, sofern Sie den/die ursprünglichen Autor(en) und die Quelle ordnungsgemäß nennen, einen Link zur Creative Commons Lizenz beifügen und angeben, ob Änderungen vorgenommen wurden.

Die in diesem Artikel enthaltenen Bilder und sonstiges Drittmaterial unterliegen ebenfalls der genannten 
Creative Commons Lizenz, sofern sich aus der Abbildungslegende nichts anderes ergibt. Sofern das betreffende Material nicht unter der genannten Creative Commons Lizenz steht und die betreffende Handlung nicht nach gesetzlichen Vorschriften erlaubt ist, ist für die oben aufgeführten Weiterverwendungen des $\mathrm{Ma}$ terials die Einwilligung des jeweiligen Rechteinhabers einzuholen.

Weitere Details zur Lizenz entnehmen Sie bitte der Lizenzinformation auf http://creativecommons.org/ licenses/by/4.0/deed.de.

\section{Literatur}

1. Arya R, Mangano FT, Horn PS et al (2013) Adverse events related to extraoperative invasive EEG monitoring with subdural grid electrodes: $\mathrm{A}$ systematic review and meta-analysis. Epilepsia 54:828-839. https://doi.org/10.1111/epi.12073

2. Baker GA, Jacoby A, Buck D et al (1997) Quality of life of people with epilepsy: a European study. Epilepsia 38:353-362. https://doi.org/10.1111/j. 1528-1157.1997.tb01128.x

3. Barbaro MF, Chesney K, Kramer DR et al (2020) Dual responsive neurostimulation implants for epilepsy. J Neurosurg 132:225-231. https://doi. org/10.3171/2018.8.jns 181362

4. Bonini F, McGonigal A, Scavarda D et al (2017) Predictive factors of surgical outcome in frontal lobe epilepsy explored with stereoelectroencephalography. Neurosurgery 83:217-225. https:// doi.org/10.1093/neuros/nyx342

5. Bonini F, McGonigal A, Trébuchon A et al (2014) Frontal lobe seizures: from clinical semiology to localization. Epilepsia 55:264-277. https://doi. org/10.1111/epi.12490

6. Busch RM, Floden DP, Ferguson L et al (2017) Neuropsychological outcome following frontal lobectomy for pharmacoresistant epilepsy in adults. Neurology 88:692-700. https://doi.org/10. 1212/wnl.0000000000003611

7. Caciagli L, Bernhardt BC, Hong S-J et al (2014) Functional network alterations and their structural substrate in drug-resistantepilepsy. Front Neurosci Switz 8:411. https://doi.org/10.3389/fnins.2014. 00411

8. Cossu G, Lebon S, Seeck M et al (2018) Periinsular anterior quadrantotomy: technical note. J Neurosurg Pediatr 21:124-132. https://doi.org/10.3171/ 2017.8.peds 17339

9. Cossu M, Fuschillo D, Casaceli G et al (2015) Stereoelectroencephalography-guided radiofrequency thermocoagulation in the epileptogenic zone: a retrospective study on 89 cases. J Neurosurg 123:1358-1367. https://doi.org/10.3171/2014.12. jns141968

10. Cuisenier P, Testud B, Minotti L et al (2021) Relationship between direct cortical stimulation and induced high-frequency activity for language mapping during SEEG recording. J Neurosurg 134:1251-1261. https://doi.org/10.3171/2020.2. jns192751

11. Delev D, Quesada CM, Grote A et al (2018) A multimodal concept for invasive diagnostics and surgery based on neuronavigated voxelbased morphometric MRI postprocessing data in previously nonlesional epilepsy. J Neurosurg 128:1178-1186. https://doi.org/10.3171/2016.12. jns161676

12. Delev D, Send K, Malter M et al (2015) Role of subdural interhemispheric electrodes in

\section{Invasive diagnostics and resective surgery in frontal lobe epilepsy}

Frontal lobe epilepsy is the most common extratemporal epilepsy syndrome representing the second most common focal epilepsy after temporal lobe epilepsy. Due to its large anatomical area and involvement of different functional zones, such as the supplementary motor area, Broca's area and premotor cortex, the semiology of frontal lobe seizures can represent a diagnostic challenge. Anatomical connections to the insula or corpus callosum may hamper the electrophysiological localization of the seizures as well. Additionally, the frequent absence of a visible epileptogenic lesion, makes the implantation of deep and/or subdural electrodes as part of the presurgical diagnostics indispensable in many cases. The spectrum of surgical resections in the frontal lobe ranges from simple focal or extended lesionectomy to frontal lobe disconnection and frontal lobectomy. The postoperative seizure outcome differs immensely, with seizure-free rates ranging from $20 \%$ to $70 \%$. The present article presents a review of invasive work-up and surgical strategies for frontal lobe epilepsy. In addition, postoperative results and factors influencing seizure outcome are summarized to point out that frontal lobe epilepsy remains a surgical challenge that may be better solved by future advances in imaging or neuromodulation.

\section{Keywords}

Drug-resistant $\cdot$ MRI negative $\cdot$ Outcome $\cdot$ Predictors $\cdot$ Neuromodulation

presurgical evaluation of epilepsy patients. World Neurosurg 84:1719-1725.e1. https://doi.org/10. 1016/j.wneu.2015.07.034

13. Delev D, Send K, Wagner J et al (2014) Epilepsy surgery of the rolandicand immediate perirolandic cortex: surgical outcome and prognostic factors. Epilepsia 55:1585-1593. https://doi.org/10.1111/ epi.12747

14. Englot DJ, Wang DD, Rolston JD et al (2012) Rates and predictors of long-term seizure freedom after frontal lobe epilepsy surgery: a systematic review and meta-analysis: clinical article. J Neurosurg 116:1042-1048. https://doi.org/10.3171/2012.1. jns111620

15. Foit NA, Bernasconi A, Bernasconi N (2020) Functional networks in epilepsy presurgical evaluation. Neurosurg Clin N Am 31:395-405. https://doi.org/10.1016/j.nec.2020.03.004

16. Gavaret M, McGonigal A, Badier J-M, Chauvel P (2004) Chapter 41 Physiology of frontal lobe seizures: pre-ictal, ictal and inter-ictal relationships. SupplClin Neurophys 57:400-407.https://doi.org/ 10.1016/s1567-424x(09)70377-0

17. Goldberg-Stern H, Gadoth N, Cahill W, Privitera M (2004) Language dysfunction after frontal lobe partial seizures. Neurology 62(9):1637-8. https:// doi.org/10.1212/01.wnl.0000123091.64548.6c. PMID: 15136702

18. Grande KM, Ihnen SKZ, Arya R (2020) Electrical stimulation mapping of brain function: a comparison of subdural electrodes and stereo-EEG. Front Hum Neurosci 14:611291. https://doi.org/ 10.3389/fnhum.2020.611291

19. Hong S-J, Bernhardt BC, Schrader DS et al (2016) Whole-brain MRI phenotyping in dysplasia-related frontal lobe epilepsy. Neurology 86:643-650. https://doi.org/10.1212/wnl.0000000000002374

20. Jobst BC, Kapur R, Barkley GL et al (2017) Brain-responsive neurostimulation in patients with medically intractable seizures arising from eloquent and other neocortical areas. Epilepsia 58:1005-1014. https://doi.org/10.1111/epi.13739

21. Jobst BC, Siegel AM, Thadani VM et al (2000) Intractable seizures of frontal lobe origin: clinical characteristics, localizing signs, and results of surgery. Epilepsia 41:1139-1152. https://doi.org/ 10.1111/j.1528-1157.2000.tb00319.x

22. Joseph Samuel P, Menon RN, Chandran A et al (2019) Seizure outcome and its predictors after frontal lobe epilepsy surgery. Acta Neurol Scand 140:259-267.https://doi.org/10.1111/ane.13139

23. Kamalboor $\mathrm{H}$, Alhindi $\mathrm{H}$, Alotaibi $\mathrm{F}$ et al (2020) Frontal disconnection surgery for drug-resistant epilepsy: outcome in a series of 16 patients. Epilepsia Open 5:475-486. https://doi.org/10. 1002/epi4.12424

24. Kasasbeh AS, Yarbrough CK, Limbrick DD et al (2012) Characterization of the supplementary motor area syndrome and seizure outcome after medial frontal lobe resections in pediatric epilepsy surgery. Neurosurgery 70:1152-1168. https://doi. org/10.1227/neu.0b013e31823f6001

25. Kellett MW, Smith DF, Baker GA, Chadwick DW (1997) Quality of life after epilepsy surgery. J Neurol Neurosurg Psychiatry 63:52. https://doi.org/10. 1136/jnnp.63.1.52

26. Larivière $S$, Bernasconi $A$, Bernasconi $N$, Bernhardt BC (2021) Connectome biomarkers of drugresistant epilepsy. Epilepsia 62:6-24. https://doi. org/10.1111/epi.16753

27. Lazow SP, Thadani VM, Gilbert KL et al (2012) Outcome of frontal lobe epilepsy surgery. Epilepsia 53:1746-1755. https://doi.org/10.1111/j.15281167.2012.03582.x

28. von Lehe M, Wagner J, Wellmer J et al (2012) Epilepsy surgery of the cingulate gyrus and the frontomesial cortex. Neurosurgery 70:900-910. https://doi.org/10.1227/neu.0b013e318237aaa3

29. Lisa F, Margaret M, Alexander W et al (2021) Cognitive outcomes following frontal lobe resection for treatment of epilepsy in children and adolescents. Epilepsy Behav 124:108265. https:// doi.org/10.1016/j.yebeh.2021.108265

30. Moeller F, Tyvaert L, Nguyen DK et al (2009) EEGfMRI. Neurology 73:2023-2030. https://doi.org/ 10.1212/wnl.0b013e3181c55d17

31. Munari C, Tassi L, Leo MD et al (1995) Videostereo-electroencephalographic investigation of 
orbitofrontal cortex. Ictal electroclinical patterns. Adv Neurol 66:273-295

32. Ramantani G, Kadish NE, Mayer H et al (2017) Frontal lobe epilepsy surgery in childhood and adolescence: Predictors of long-term seizure freedom, overall cognitive and adaptive functioning. Neurosurgery 83:93-103. https://doi.org/10. 1093/neuros/nyx340

33. Rennert RC, Khan U, Tatter SB et al (2018) Patterns of clinical use of stereotactic laser ablation: analysis of a multicenter prospective registry. World Neurosurg 116:e566-e570. https://doi.org/ 10.1016/j.wneu.2018.05.039

34. Rincon N, Barr D, Velez-Ruiz N (2021) Neuromodulation in drug resistant epilepsy. Aging Dis 12:1070-1080. https://doi.org/10.14336/ad.2021. 0211

35. Roper SN (2009) Surgical treatment of the extratemporal epilepsies. Epilepsia 50:69-74. https://doi.org/10.1111/j.1528-1167.2009.02239. $x$

36. Rosenow F, Lüders H (2001) Presurgical evaluation of epilepsy. Brain 124:1683-1700. https://doi.org/ 10.1093/brain/124.9.1683

37. Salanova V, Sperling MR, Gross RE et al (2021) The SANTÉ study at 10 years offollow-up: effectiveness, safety, and sudden unexpected death in epilepsy. Epilepsia 62:1306-1317. https://doi.org/10.1111/ epi.16895

38. Sander JW, Shorvon SD (1996) Epidemiology of the epilepsies. J Neurol Neurosurg Psychiatry 61:433-443. https://doi.org/10.1136/jnnp.61.5. 433

39. Sebastian B, Hajo MH (2012) Chapter 15 Extratemporal epilepsies. In: Stefan H, William TH (Hrsg) Epilepsy. Elsevier, , S241-256

40. Unnwongse $\mathrm{K}$, Wehner T, Foldvary-Schaefer N (2012) Mesial frontal lobe epilepsy. J Clin Neurophysiol 29:371-378. https://doi.org/10. 1097/wnp.0b013e31826b3c60

41. Veersema TJ, Ferrier CH, van Eijsden P et al (2017) Seven tesla MRI improves detection offocal cortical dysplasia in patients with refractory focal epilepsy. Epilepsia Open 2:162-171. https://doi.org/10. 1002/epi4.12041

42. Wen HT, Róz LMD, Rhoton AL et al (2017) Frontal lobe decortication (frontal lobectomy with ventricular preservation) in epilepsy-part 1: anatomic landmarks and surgical technique. World Neurosurg 98:347-364. https://doi.org/10.1016/j. wneu.2016.10.090

43. Wiebe S (2012) Outcome patterns in epilepsy surgery - the long-term view. Nat Rev Neurol 8:123-124. https://doi.org/10.1038/nrneurol. 2012.9

44. Wiebe S, Blume WT, Girvin JP et al (2001) A randomized, controlled trial of surgery for temporal-lobe epilepsy. NEngl JMed 345:311-318. https://doi.org/10.1056/nejm200108023450501

45. Zhao B, Zhang C, Wang X et al (2020) Orbitofrontal epilepsy: distinct neuronal networks underlying electroclinical subtypes and surgical outcomes. J Neurosurg. https://doi.org/10.3171/2020.5. jns20477

\section{Fe Strack, Xiaying Lin, Martina Schlegl}

\section{Wackelkontakt: Epilepsie bei Kindern leicht erklärt}

\section{Göttingen - Bern - Wien: Hogrefe Verlag 2021, 64 S., (ISBN: 978-3-456-86197-5), Hardcover 24,95 EUR}

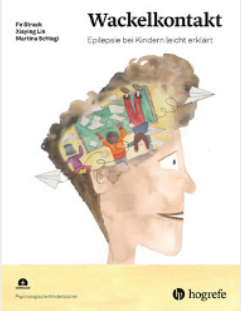

Dieses Buch ist Teil der seit 2017 erscheinenden Reihe „Psychologische Kinderbücher" der Hogrefe-Verlagsgruppe mit zuvor schon 16 anderen und geplanten weiteren Bänden. Diese richten sich direkt an Kinder mit eigenen gesundheitlichen Störungen oder psychischen und sozialen Problemen auch anderer Familienmitglieder, führen in den jeweiligen Problembereich ein und liefern Tipps und Tricks zu deren Bewältigung für die Kinder und ihre Eltern sowie sonstige Angehörige. Jedes Buch beginnt mit einer Geschichte, in der die Protagonistin oder der Protagonist lernt, mit seinen Ängsten und Sorgen umzugehen, um sie am Ende zu überwinden. Danach sind die kleinen Leserinnen und Leser selber am Zug und lernen sich anhand bunter Mitmachseiten besser kennen. Weiter folgen Informationen zu den Belastungen für Eltern und schließlich weiterführende Adressen und Kontakte.

Die drei jungen, zwischen 1986 und 1993 geborenen Autorinnen stammen alle aus Deutschland (Xiaying Lin ursprünglich aus (hina), alle kamen entweder in der Familie oder in der Schule in Kontakt mit Epilepsie. Die Texterinnen (Xiaying Lin und Martina Schlegl) beschäftigten sich während ihres Psychologiestudiums insbesondere mit der Kinder- und Jugendpsychologie. Die Illustratorin (Fe Strack) hat einen Bruder mit Epilepsie,

Der kleine Jojo hat Epilepsie und bekommt beim Sportunterricht einen Anfall, weshalb er ins Krankenhaus kommt. Danach ist zunächst einmal alles anders als zuvor, Joho geht nicht mehr gerne in die Schule, auch weil die anderen Kinder sich auch noch lustig über inn machen. Jojo erklärt zunächst seiner Freundin Charly, was es mit Epilepsie auf sich hat. Dabei machen viele Nervenzellen im Gehirn "gleichzeitig denselben Quatsch", weshalb bei inm für kurze Zeit manches an- ders läuft als es eigentlich soll. Dann haben Charly und Jojo eine geniale Idee. Mit Unterstützung ihrer Klassenlehrerin bekommen sie die anderen Kinder in der Klasse dazu, alle gleichzeitig mehr oder weniger wirr durcheinander zu reden. Dann erklären sie ihnen, dass dies dasselbe ist, was bei einem Anfall in Jojos Gehirn geschieht.

Im nächsten Teil des Buches finden sich leicht verständliche, kurze medizinische Informationen über die verschiedenen Formen epileptischer Anfälle sowie die Untersuchungsund Behandlungsmöglichkeiten inklusive einer - sofern erforderlich - psychologischen Mitbetreuung. Darauf folgen Tipps für den Alltag - u.a. mit dem Hinweis auf ausreichend Schlaf oder Anleitungen zum (Nach-) Spielen von Geschichten oder zum richtigen Verhalten bei Anfällen („Das Notfallkommando“). Am Ende finden sich Hinweise zu den „Mach mit"- Seiten sowie nützlichen Adressen und Kontakten. Diese können nach erfolgter Registrierung mit einem entsprechenden Code über einen Internet-Link heruntergeladen werden und auf dem eigenen Computer gespeichert werden.

Insgesamt ein sehr empfehlenswertes Buch für Kinder mit Epilepsie und deren Geschwister im Alter zwischen etwa sechs und zehn Jahren. Die Ausstattung und der durchgehend vierfarbige Druck des großformatigen Buches sind sehr gut, der Preis noch akzeptabel. Mein Glückwunsch an den Verlag zu dem mutigen und gelungenen Projekt.

Günter Krämer, Zürich 